\title{
Leptin secretory dynamics and associated disordered eating psychopathology across the weight spectrum
}

\author{
Charumathi Baskaran 1,2, Kamryn T Eddy ${ }^{3}$, Karen K Miller², Erinne Meenaghan², \\ Madhusmita Misra ${ }^{1,2}$ and Elizabeth A Lawson ${ }^{2}$ \\ ${ }^{1}$ Pediatric Endocrine Unit, Massachusetts General Hospital for Children and Harvard Medical School, 101 Merrimac, \\ Suite 615, Boston, Massachusetts 02114, USA, ${ }^{2}$ Neuroendocrine Unit and ${ }^{3}$ Eating Disorder Clinical Research \\ Program, Massachusetts General Hospital for Children and Harvard Medical School, Boston, Massachusetts, USA
}

Correspondence

should be addressed

to C Baskaran

Email

cbaskaran@mgh.harvard.edu

\begin{abstract}
Objective: Leptin secretory dynamics across the weight spectrum and their relationship with disordered eating psychopathology have not been studied. Our objective was to compare leptin secretory dynamics in 13 anorexia nervosa (AN), 12 overweight/obese (OB) and 12 normal-weight women using deconvolution analysis.

Methods: In this cross-sectional study conducted at a tertiary referral center, serum leptin levels were obtained every 20 min from 2000 to $0800 \mathrm{~h}$. Dual energy X-ray absorptiometry was used to measure percent body fat. Disordered eating psychopathology was assessed by the Eating Disorders Examination-Questionnaire (EDE-Q) and the Eating Disorders Inventory-2 (EDI-2).

Results: The groups differed for basal leptin secretion (BASAL) $(P=0.02)$. Mean leptin pulse amplitude, pulse mass, total pulsatile secretion (TPS) and area under the curve (AUC) were significantly different between groups before and after adjustment for BASAL ( $P<0.0001$ for all). Leptin AUC correlated strongly with TPS $(r=0.97, P<0.0001)$ and less with BASAL $(r=0.35, P=0.03)$. On multivariate analysis, only TPS was a significant predictor of leptin AUC $(P<0.0001)$. TPS was inversely associated with most EDE-Q and EDI-2 parameters and the associations remained significant for EDE-Q eating concern $(P=0.01)$, and EDI-2 asceticism, ineffectiveness and social insecurity $(P<0.05)$ after adjusting for BASAL. These relationships were not significant when controlled for percent body fat.

Conclusion: Secretory dynamics of leptin differ across weight spectrum, with mean pulse amplitude, mean pulse mass and TPS being low in AN and high in OB. Pulsatile, rather than basal secretion, is the major contributor to leptin AUC. Decreased pulsatile leptin is associated with disordered eating psychopathology, possibly reflecting low percent body fat in AN.
\end{abstract}

\section{Introduction}

Leptin, an anorexigenic hormone that is primarily produced by adipocytes, plays a major role in the regulation of food intake and energy homeostasis. Leptin is secreted in a pulsatile fashion (1) and levels of leptin are strongly associated with percent body fat (2), such that leptin levels are decreased in patients with low weight conditions such as anorexia nervosa (AN) (3) and elevated in high weight states such as obesity (4). Nutritional and hormonal factors are known to regulate leptin secretion; (c) 2016 European Society of Endocrinology Printed in Great Britain however, it is unclear how these mechanisms impact basal vs pulsatile leptin secretion. Glucose and amino acid containing foods are the primary stimuli for leptin secretion and may account for its pulsatility $(5,6)$. In contrast, fatty acids inhibit leptin secretion (7). Additionally, endogenous hormones such as basal insulin and glucocorticoids are strong stimuli for leptin secretion. However, very little is known regarding the pattern of secretion of this hormone across the weight spectrum.

Published by Bioscientifica Ltd 
Leptin acts in conjunction with other appetite regulating hormones such as ghrelin, peptide YY (PYY) and cortisol to regulate food intake. Of these, ghrelin and cortisol stimulate appetite while leptin and PYY are anorexigenic. Although relationships between levels of leptin and these hormones have been described $(8,9,10)$, data evaluating the associations between leptin secretory patterns and appetite regulating hormones are limited to our study in adolescents (11), and have not been reported in adults. Leptin levels are known to vary with pubertal status due to the combined influence of the divergent effects of fat and lean mass accumulation and the varying testosterone levels during puberty, resulting in girls having an increase and boys having a decrease in leptin levels as puberty progresses (12). However, there are no data regarding secretory patterns of leptin in adult women with AN in whom the impact of pubertal status no longer exists. Additionally, although one study examined leptin secretory patterns in overweight/obese (OB) women with polycystic ovarian syndrome (13), a condition in which variable leptin levels have been reported $(14,15)$, data on leptin secretory patterns in healthy $\mathrm{OB}$ women with regular menses are limited.

Disordered eating behavior is often seen across the weight spectrum and has been shown in one earlier study to be associated with leptin levels regardless of body weight (16) suggesting that eating behaviors may affect leptin secretion. Our group previously reported an inverse association of leptin levels with several measures of disordered eating assessed by eating disorder questionnaires, however these relationships were not significant when controlling for body weight (17). Assessment of leptin secretory dynamics would allow for a more in depth examination of the relationship between leptin dynamics, including basal and pulsatile secretion as well as integrated leptin levels, and disordered eating measures, and may improve our understanding of these associations with respect to weight status.

We therefore used state-of-the-art deconvolution analysis to examine leptin secretory dynamics and its relationship to disordered eating psychopathology in AN, healthy normally menstruating OB and healthy normalweight women. We hypothesized that leptin secretory parameters would differ between groups, demonstrating the mechanism for low leptin levels in AN and high levels in $\mathrm{OB}$ women compared with normal-weight controls. We also hypothesized that secretory parameters of leptin would be inversely associated with severity of eating disorder thoughts and behaviors.

\section{Subjects and methods}

We evaluated the secretory patterns of leptin in 37 adult women; 13 with AN, 12 OB women and 12 normal weight women (C) using deconvolution analysis. Subject characteristics, hormone levels, and Eating Disorders Examination-Questionnaire (EDE-Q) and Eating Disorders Inventory-2 (EDI-2) scores have been previously reported $(17,18,19,20,21)$. Analysis of leptin secretory parameters and their relationship with measures of disordered eating psychopathology and other appetite-regulating hormones have not been published and are presented in this report.

All participants were between ages 18 and 45 years. Inclusion criteria for AN participants included a DSM IV criteria of a weight $<85 \%$ of ideal body weight (IBW) associated with an intense fear of gaining weight, an impaired body image and amenorrhea for at least 3 consecutive months preceding study participation. OB controls were required to have a BMI between 25 and $40 \mathrm{~kg} / \mathrm{m}^{2}$, and normal weight controls were at least $90 \%$ of IBW with a BMI $<25 \mathrm{~kg} / \mathrm{m}^{2}$. Both control groups were otherwise healthy with normal menstrual cycles and no significant medical or psychiatric disease. Subjects with abnormal thyroid function tests, diabetes mellitus, active drug or alcohol abuse, and those who were pregnant or breastfeeding were excluded from the study.

The study was approved by Partners Human Research Committee. Informed consent was obtained prior to any procedures. At a screening visit, height and weight were obtained, medical history and physical exam were performed, and labs were drawn. Eligible participants were admitted to the Clinical Research Center overnight for the study procedures. \%IBW and BMI were reevaluated at this visit. Dual energy X-ray absorptiometry was performed to assess body composition. Disordered eating psychopathology was assessed by administering EDE-Q and EDI-2 questionnaires. An IV catheter was placed by $1800 \mathrm{~h}$ and subjects were allowed to acclimate to the environment. Serum was drawn every $20 \mathrm{~min}$ from $2000 \mathrm{~h}$ to $0800 \mathrm{~h}$. Subjects were asked to start fasting at $2000 \mathrm{~h}$. Serum was pooled for integrated measures of $12 \mathrm{~h}$ cortisol. Fasting ghrelin, PYY, insulin, insulin-like growth factor 1 (IGF1) and estradiol $\left(\mathrm{E}_{2}\right)$ levels were obtained at $0745 \mathrm{~h}$.

\section{Assay and biochemical analysis}

All blood samples were frozen and stored at $-80{ }^{\circ} \mathrm{C}$ until analysis. Serum leptin was measured using a radioimmunoassay by Linco Research, St Charles, MS. The intra-assay coefficient of variation $(\mathrm{CV})$ ranged from 5.2 to 
$7.5 \%$ and inter-assay variation from 3.2 to $8.9 \%$. The minimum detectable concentration was $0.1 \mathrm{ng} / \mathrm{ml}$. Serum cortisol was measured by a chemiluminescent micro particle immunoassay (Architect System, Abbot Diagnostics, Abbott Park, IL, USA) with an intra-assay CV of 2.1$4.8 \%$ and sensitivity of $0.8 \mathrm{mcg} / \mathrm{dl}$. An RIA kit from Linco Research, a division of Millipore Inc., was used to assess plasma ghrelin levels (intra-assay CV 10.0-14.4\% and inter-assay CV $14.7-16.7 \%$, sensitivity $93 \mathrm{pg} / \mathrm{ml}$ ) and serum PYY (intra-assay CV $1.5-2.7 \%$ and inter-assay CV $6.1-6.9 \%$, sensitivity $1.4 \mathrm{pg} / \mathrm{ml}$ ). Serum insulin was measured using an ultrasensitive assay (Access Immunoassay Systems, Beckman Coulter, Brea, CA, USA) which had an intra-assay CV of $2.0-4.2 \%$, inter-assay CV of 3.1-5.6\% and a sensitivity of $0.03 \mu \mathrm{IU} / \mathrm{ml}$. Fasting serum IGF1 levels were measured by chemiluminescent immunometric assay (Immulite 2000; Diagnostics Products Corp., Los Angeles, CA, USA) (intra-assay CV 2.3-3.9\%, sensitivity $20 \mathrm{ng} / \mathrm{ml}) . E_{2}$ was measured by chemiluminescent immunoassay (Access Immunoassay Systems, Beckman Coulter) (intra-assay CV 2.4-4.2\%, inter-assay CV 4.4-8.2\% and sensitivity $20 \mathrm{pg} / \mathrm{ml}$ ).

Deconvolution analysis - Leptin levels obtained by frequent sampling, every $20 \mathrm{~min}$, were analyzed using Deconv feature of the Autodecon software. The software can be downloaded online from: 'https://www.researchgate.net/publication/262566415_AutoDecon_and_Pulse_ XP_Software?ev=prf_pub.' Deconvolution analysis is a mathematical algorithm based software that provides information regarding basal secretory rate, half-life, number of pulses, pulse interval, mean pulse amplitude, mean pulse mass and total area under the curve (AUC) based on levels obtained from frequent sampling (22). As in our study in adolescent $\mathrm{AN}$, we applied a half life of $(24.9 \pm 4.4 \mathrm{~min})$ to the model as determined by Klein et al. (23). Total basal secretion (BASAL) was then calculated by multiplying the basal secretory rate $(\mathrm{ng} / \mathrm{ml}$ per min) with the total duration of blood draw (720 min). Total pulsatile secretion (TPS) was calculated by multiplying mean pulse mass by the total number of pulses over the duration of frequent sampling. The sum of BASAL and TPS provided the total leptin secretion over $12 \mathrm{~h}$.

Disordered eating questionnaires $~ E D E-Q$ is a selfreported measure assessing the severity of eating disorder psychopathology in four categories: i) dietary restraint, ii) eating concern, iii) shape concern and iv) weight concern. A global score can be calculated to render a dimensional assessment of eating disorder psychopathology (24). Normative data (mean \pm s.D.) for EDE-Q measures were presented in our earlier report (17) based on prior studies in adult women $(25,26)$ and are as follows: dietary restraint, $1.3 \pm 1.4$ (25), $1.62 \pm 1.54$ (26); eating concern, $0.76 \pm 1.06,1.11 \pm 1.11$; weight concern, $1.79 \pm 1.51,1.97 \pm 1.56$; shape concern, $2.23 \pm 1.65,2.27 \pm$ 1.54; and global concern, $1.52 \pm 1.25,1.74 \pm 1.30$.

The EDI-2 is a self report questionnaire with 91 items and 11 subscales (27). It is a validated measure used to assess drive for thinness, bulimia, body dissatisfaction, ineffectiveness, perfectionism, interpersonal distrust, interoception, maturity fears, asceticism, impulse regulation and social insecurity. The normative data (mean \pm s.E.M. of measurement) for EDI- 2 are based on the EDI manual norms (27) and are as follows: drive for thinness, $5.5 \pm 2.2$; bulimia, $1.2 \pm 0.9$; body dissatisfaction, $12.2 \pm 3.0$; ineffectiveness, $2.3 \pm 1.6$; perfectionism, $6.2 \pm$ 2.5 , interpersonal distrust, $2.0 \pm 1.3$; interoceptive awareness, $3.0 \pm 2.1$; maturity fears, $2.7 \pm 1.3$; asceticism, $3.4 \pm$ 1.6; impulse regulation, $2.3 \pm 1.6$; and social insecurity, $3.3 \pm 1.5$.

\section{Statistical analysis}

JMP Pro 11 was used for statistical analysis (SAS Institute, Cary, NC, USA). Descriptive data are presented as mean \pm S.D. The Shapiro-Wilk test was used to determine whether or not data were normally distributed. Continuous variables were assessed using ANOVA. The Tukey Kramer test was used to compare differences between any two groups while accounting for multiple comparisons. For non-parametric data, the Kruskal-Wallis test was used to compare group differences, followed by the Steel Dwass test to control for multiple comparisons. As leptin secretory parameters were not normally distributed, we used non-parametric tests (Spearman correlation) to evaluate associations of leptin secretion with other hormones and with disordered eating psychopathology. Multivariate analysis was performed to assess associations of leptin TPS with other hormones and disordered eating psychopathology after adjusting for total BASAL and percent body fat obtained by DEXA. A $P$ value of $<0.05$ was considered statistically significant.

\section{Results}

\section{Clinical characteristics}

The participant characteristics and hormonal parameters are summarized in Table 1. As per study design, the BMI 
Table 1 Participant characteristics and hormonal parameters across the groups.

\begin{tabular}{|c|c|}
\hline & $\mathbf{A N}(n=13)$ \\
\hline Age (years) & $26.6 \pm 6.5$ \\
\hline Baseline BMI $\left(\mathrm{kg} / \mathrm{m}^{2}\right)^{\mathrm{a}}$ & $18.5 \pm 0.9$ \\
\hline Percent body fat $(\%)^{b}$ & $18 \pm 4$ \\
\hline Fasting ghrelin $(\mathrm{pg} / \mathrm{ml})^{c}$ & $1126 \pm 372$ \\
\hline $12 \mathrm{~h}$ pooled cortisol $(\mu \mathrm{g} / \mathrm{ml})^{\mathrm{d}}$ & $11.3 \pm 3.3$ \\
\hline Fasting PYY $(\mathrm{pg} / \mathrm{ml})^{\mathrm{e}}$ & $97 \pm 27$ \\
\hline *Fasting insulin $(\mu \mathrm{IU} / \mathrm{ml})^{f}$ & $2.6 \pm 1.4$ \\
\hline Fasting IGF1 (ng/ml) & $174 \pm 73$ \\
\hline *Estradiol $(\mathrm{pg} / \mathrm{ml})^{\mathrm{g}}$ & $36 \pm 18$ \\
\hline
\end{tabular}

\begin{tabular}{c}
\hline $\mathbf{C}(n=12)$ \\
\hline $28.4 \pm 6.3$ \\
$22.0 \pm 1.5$ \\
$26 \pm 5$ \\
$1006 \pm 324$ \\
$7.8 \pm 1.4$ \\
$80 \pm 24$ \\
$4.6 \pm 1.7$ \\
$234 \pm 62$ \\
$53 \pm 25$ \\
\hline
\end{tabular}

\begin{tabular}{c}
\hline OB $(n=12)$ \\
\hline $28.5 \pm 8.2$ \\
$31.3 \pm 4.1$ \\
$37 \pm 4$ \\
$539 \pm 195$ \\
$5.9 \pm 1.8$ \\
$66 \pm 8$ \\
$7.7 \pm 3.4$ \\
$243 \pm 112$ \\
$68 \pm 53$ \\
\hline
\end{tabular}

\begin{tabular}{c}
\hline P value \\
\hline NS \\
$<\mathbf{0 . 0 0 0 1}$ \\
$<\mathbf{0 . 0 0 0 1}$ \\
$\mathbf{0 . 0 0 0 2}$ \\
$<\mathbf{0 . 0 0 0 1}$ \\
$\mathbf{0 . 0 0 6}$ \\
$<\mathbf{0 . 0 0 0 1}$ \\
0.11 \\
$\mathbf{0 . 0 2}$
\end{tabular}

* $P$ value obtained by Wilcoxon and Steel Dwass used for multiple comparisons.

OB vs $A N$ and $C P<0.0001$, AN vs $C P=0.004$.

'OB vs $A N$ and $C P<.0001$, AN vs $C P=0.0001$.

'OB vs $A N$ and $C P<0.01$.

OB vs AN $P<0.0001$ AN vs $C P=0.003$.

OOB vs AN $P=0.004$ a-e $P$ value obtained by ANOVA and Tukey Kramer used for multiple comparisons.

'OB vs $A N$ and $C P \leq 0.02$, AN vs $C P=0.02$.

${ }^{9} \mathrm{OB}$ vs $\mathrm{AN} P=0.04$.

and percent body fat of the subjects were significantly different among the three groups. Fasting ghrelin, $12 \mathrm{~h}$ pooled cortisol and PYY values were highest in the AN group and lowest in the OB group $(P<0.01)$.

\section{Deconvolution analysis}

Leptin secretory parameters obtained from deconvolution analysis are shown in Table 2. Figure 1 depicts leptin secretory patterns obtained using deconvolution analysis in one representative subject from each group. The number of peaks, half-life and pulse interval were similar in the $\mathrm{AN}, \mathrm{OB}$ and control groups. Leptin AUC, mean pulse amplitude, mean pulse mass, TPS and total secretion were lowest in AN and highest in OB groups $(P<0.0001)$. Leptin BASAL was significantly higher in the $\mathrm{OB}$ group compared with the AN group $(P=0.04)$, but did not differ when $\mathrm{AN}$ or $\mathrm{OB}$ groups were compared with normalweight controls. Because there was a wide range of age for study participants (18-45 years), we divided subjects into two age groups based on the median age of 26 years. There were no significant differences in leptin secretory parameters between those who were 26 years old or younger compared with those over 26 years old.

Leptin AUC correlated strongly with TPS $(r=0.97$, $P<0.0001)$ and less so with BASAL $(r=0.35, P=0.03)$. On multivariate analysis, only TPS was a significant predictor of leptin AUC $(P<0.0001)$ indicating that TPS was the major contributor to leptin AUC. In order to assess the impact of BASAL vs TPS on other hormones and disordered eating psychopathology measures, we performed multivariate analysis controlling for BASAL.

\section{Leptin secretory dynamics and body fat}

Both leptin TPS $(r=0.94, P<0.0001)$ and AUC $(r=0.95$, $P<0.0001$ ) were strongly associated with percent body fat. In contrast, the correlation between BASAL and percent

Table 2 Leptin secretory dynamics across the groups. Overall $P$ value obtained using the Kruskall-Wallis test. $P$ values for differences between any two groups after adjusting for multiple comparisons were obtained using the Steel-Dwass test.

Number of peaks in $12 \mathrm{~h}$

Half-life (minutes)

Pulse interval (minutes)

AUC (ng/ml per $12 \mathrm{~h})$

Mean pulse amplitude $(\mathrm{ng} / \mathrm{ml})$

Mean pulse mass $(\mathrm{ng} / \mathrm{ml})$

Total pulsatile secretion $(\mathrm{ng} / \mathrm{ml}$ per $12 \mathrm{~h}$ )

Total basal secretion $(\mathrm{ng} / \mathrm{ml}$ per $12 \mathrm{~h})$

Total leptin secretion ( $\mathrm{ng} / \mathrm{ml}$ per $12 \mathrm{~h}$ )

\begin{tabular}{c} 
AN $(n=13)$ \\
\hline $18.2 \pm 0.4$ \\
$24.56 \pm 4.42$ \\
$39.89 \pm 0.80$ \\
$3011 \pm 1986$ \\
$0.18 \pm 0.12$ \\
$4.8 \pm 3.1$ \\
$87.9 \pm 56.0$ \\
$0.08 \pm 0.09$ \\
$87.9 \pm 56.0$ \\
\hline
\end{tabular}

\begin{tabular}{ccc}
\hline $\mathbf{C}(n=12)$ & & OB $(n=12)$ \\
\cline { 1 - 1 } $18.2 \pm 0.9$ & & $18.3 \pm 0.7$ \\
$24.87 \pm 4.03$ & & $24.90 \pm 3.81$ \\
$39.33 \pm 0.89$ & & $39.93 \pm 1.44$ \\
$8897 \pm 3385$ & $24062 \pm 10359$ \\
$0.58 \pm 0.29$ & $1.40 \pm 0.72$ \\
$14.3 \pm 5.6$ & & $38.2 \pm 18.5$ \\
$263.6 \pm 110.7$ & $709.8 \pm 382.4$ \\
$0.07 \pm 0.03$ & $0.13 \pm 0.08$ \\
$263.7 \pm 110.7$ & $709.9 \pm 382.4$ \\
\hline
\end{tabular}

\begin{tabular}{c}
\hline AN vs C \\
\hline NS \\
NS \\
NS \\
0.0009 \\
0.002 \\
0.001 \\
0.001 \\
NS \\
0.001 \\
\hline
\end{tabular}

\begin{tabular}{c}
\hline OB vs C \\
\hline NS \\
NS \\
NS \\
0.0002 \\
0.0006 \\
0.0001 \\
0.0002 \\
NS \\
0.0002 \\
\hline
\end{tabular}



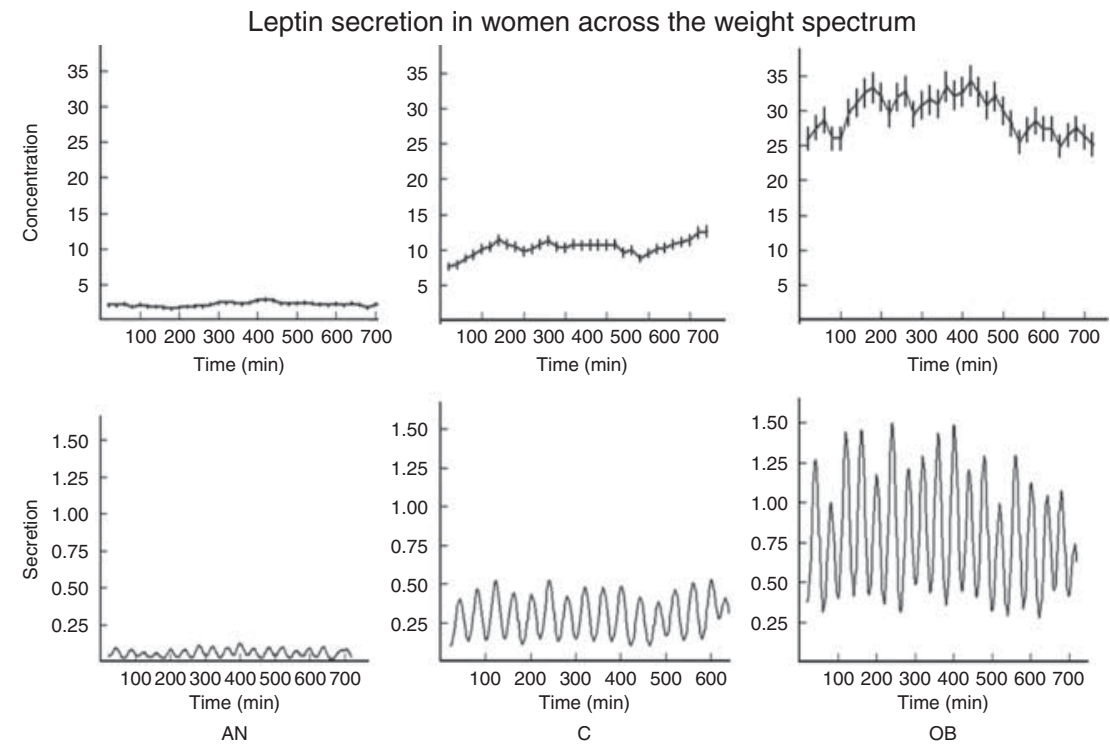

\section{Figure 1}

Deconvolution analysis. (Top panel) Depicts leptin concentration in a subject with anorexia nervosa (AN), a normal weight healthy control and an overweight/obese control (OB). (Bottom panel) Depicts the individual secretory pattern for the same individuals. Patients with AN had low pulse amplitude and

body fat was less robust $(r=0.33, P=0.05)$. Leptin TPS $(r=0.71 P<0.0001)$ but not BASAL was strongly correlated with trunk to extremity fat ratio (a surrogate for visceral fat) (28). However, both leptin TPS $(r=0.94, P<0.0001)$ and BASAL $(r=0.36, P=0.03)$ correlated with total fat mass (a surrogate for subcutaneous fat mass) (29).

\section{Leptin secretory dynamics and other hormones including appetite-regulating hormones}

Leptin TPS and AUC were inversely associated with fasting ghrelin ( $r=-0.67$ for both), 12-h pooled cortisol $(r=-0.77$ and -0.75$)$ and PYY $(r=-0.63$ and -0.59$)$ $(P<0.001$ for all). BASAL was inversely associated with PYY ( $r=-0.35, P=0.04$ ), but was unrelated to fasting ghrelin or $12 \mathrm{~h}$ pooled cortisol. After controlling for BASAL in multivariate models, TPS retained its negative associations with fasting ghrelin $(P<0.0001), 12 \mathrm{~h}$ pooled cortisol $(P<0.0001)$ and PYY $(P=0.002)$.

We performed additional analyses examining the relationship between leptin secretory parameters and hormones that could potentially impact leptin levels, such as insulin $(6,30)$, and hormones that could impact eating disorder psychopathology, such as IGF1 and $\mathrm{E}_{2}(31)$. pulse mass compared with normal weight controls $(P<0.0001)$. OB controls had higher pulse amplitude and pulse mass compared with normal weight controls $(P<0.0001)$. The unit of time for the $x$ axis is minutes.

Leptin TPS and AUC were strongly correlated with fasting insulin ( $r=0.83$ and 0.82 respectively, $P<0.0001)$ and there were less robust associations between BASAL and insulin $(r=0.35, P=0.03)$ levels. Leptin TPS was also associated with fasting IGF1 $(r=0.35, P=0.04)$ and $\mathrm{E}_{2}$ ( $r=0.53 P=0.0008$ ) levels. These associations of TPS with insulin $(P<0.0001)$ and $\mathrm{E}_{2}(P=0.01)$ persisted after controlling for BASAL. However, associations of these hormones with TPS were no longer significant after controlling for percent body fat.

\section{Leptin secretory dynamics and disordered eating measures}

Participant scores for EDI-2 and EDE-Q subscales are presented in Table 3. Most EDI-2 measures and all EDE-Q measures were elevated in AN compared with $\mathrm{C}$ and $\mathrm{OB}$. Further, TPS was negatively associated with most scales for EDI-2 including drive for thinness, ineffectiveness, interpersonal distrust, interoceptive awareness, asceticism, impulse regulation and social insecurity, and all EDE-Q subscales and global score (Table 4). BASAL was negatively associated with scores for EDI-2 bulimia, ineffectiveness, asceticism and social insecurity. Among the EDE-Q 
Table 3 EDI-2 and EDE-Q scores across the groups. Overall $P$ value was obtained using the Kruskall-Wallis test. $P$ values for differences between any two groups after adjusting for multiple comparisons were obtained using the Steel-Dwass test.

\begin{tabular}{l}
\hline \\
\hline EDI-2 \\
Drive for thinness \\
Bulimia \\
Body dissatisfaction \\
Ineffectiveness \\
Perfectionism \\
Interpersonal distrust \\
Interoceptive awareness \\
Maturity fears \\
Asceticism \\
Impulse regulation \\
Social insecurity \\
EDE-Q \\
Dietary restraint \\
Eating concern \\
Shape concern \\
Weight concern \\
Global concern
\end{tabular}

$\begin{array}{r}\multicolumn{1}{c}{\text { AN }} \\ \hline \\ 10.8 \pm 7.4 \\ 0.9 \pm 1.3 \\ 13.4 \pm 8.2 \\ 7.5 \pm 6.2 \\ 7.0 \pm 5.3 \\ 6.3 \pm 4.0 \\ 7.2 \pm 7.4 \\ 6.2 \pm 3.4 \\ 6.7 \pm 3.1 \\ 2.0 \pm 2.6 \\ 8.4 \pm 4.7 \\ 2.8 \pm 1.5 \\ 2.8 \pm 1.5 \\ 3.7 \pm 1.8 \\ 3.3 \pm 1.7 \\ 3.1 \pm 1.5 \\ \hline\end{array}$

\begin{tabular}{c}
\hline C \\
\hline $0.9 \pm 1.3$ \\
$0.3 \pm 0.7$ \\
$3.9 \pm 4.3$ \\
$0.8 \pm 1.1$ \\
$3.5 \pm 2.5$ \\
$1.1 \pm 2.0$ \\
$0.6 \pm 1.3$ \\
$3.9 \pm 1.7$ \\
$3.2 \pm 2.6$ \\
$0.4 \pm 1.3$ \\
$0.7 \pm 1.1$ \\
$0.6 \pm 0.7$ \\
$0.2 \pm 0.3$ \\
$0.8 \pm 0.7$ \\
$0.5 \pm 0.6$ \\
$0.5 \pm 0.5$ \\
\hline
\end{tabular}

\begin{tabular}{c}
\hline ОВ \\
\hline \\
$1.7 \pm 2.0$ \\
$0.3 \pm 0.8$ \\
$9.2 \pm 7.2$ \\
$0.8 \pm 1.1$ \\
$4.1 \pm 3.7$ \\
$2.2 \pm 1.9$ \\
$0.8 \pm 1.3$ \\
$4.2 \pm 1.4$ \\
$2.3 \pm 1.4$ \\
$0.2 \pm 0.6$ \\
$1.6 \pm 1.4$ \\
$0.9 \pm 1.0$ \\
$0.2 \pm 0.2$ \\
$1.4 \pm 1.2$ \\
$1.4 \pm 0.9$ \\
$0.9 \pm 0.7$ \\
\hline
\end{tabular}

\begin{tabular}{c}
\hline$P$ value \\
\hline \\
0.001 \\
NS \\
0.009 \\
0.003 \\
NS \\
0.002 \\
0.01 \\
NS \\
0.002 \\
0.01 \\
0.0002 \\
\\
0.0009 \\
$<.0001$ \\
0.0007 \\
0.0003 \\
0.0002 \\
\hline
\end{tabular}

\begin{tabular}{c}
\hline AN vs C \\
\hline \\
0.005 \\
$N S$ \\
0.02 \\
0.02 \\
$N S$ \\
0.006 \\
0.03 \\
$N S$ \\
0.03 \\
$N S$ \\
0.002 \\
\\
0.001 \\
0.0001 \\
0.002 \\
0.002 \\
0.0009 \\
\hline
\end{tabular}

\begin{tabular}{|c|c|}
\hline AN vs OB & OB vs C \\
\hline 0.01 & NS \\
\hline NS & NS \\
\hline NS & NS \\
\hline 0.01 & NS \\
\hline NS & NS \\
\hline 0.02 & NS \\
\hline NS & NS \\
\hline NS & NS \\
\hline 0.002 & NS \\
\hline 0.03 & NS \\
\hline 0.004 & NS \\
\hline 0.02 & NS \\
\hline 0.0003 & NS \\
\hline 0.01 & NS \\
\hline 0.02 & NS \\
\hline 0.005 & NS \\
\hline
\end{tabular}

measures, only dietary restraint had an association with BASAL. In multivariate analysis, TPS continued to be significantly associated with EDI-2 measures of asceticism, ineffectiveness and social insecurity $(P<0.05)$ and the EDE-Q measure of eating concern $(P=0.01)$ after adjusting for BASAL. However, all associations were lost when percent body fat was included in the model.

\section{Discussion}

To our knowledge, this is the first study comparing secretory dynamics of leptin in women across the weight spectrum, and examining the relationship between leptin secretion and i) appetite regulating hormones, as well as ii) disordered eating psychopathology. We show that leptin basal and pulsatile secretory parameters are low in AN and high in OB women. Next, we demonstrate that pulsatile leptin secretion is the major contributor to leptin AUC across the weight spectrum and is negatively associated with levels of appetite-regulating hormones cortisol, ghrelin and PYY. Finally, our findings demonstrate that lower pulsatile leptin secretion is associated with greater severity of disordered eating thoughts and behaviors as assessed by the EDI-2 and EDE-Q.

Although leptin secretory dynamics have been previously described in adolescent girls with $\mathrm{AN}$, healthy and $\mathrm{OB}$ men, and $\mathrm{OB}$ women with PCOS, leptin secretory patterns have not been previously explored across adult women with AN, normal-weight women and OB women with regular menses. Examining the differences in secretory patterns across the weight spectrum in a sex specific manner is all the more important given that women in general have $40 \%$ higher leptin levels compared with men (32). Here we report that adult women with AN have lower leptin pulse amplitude, pulse mass, TPS and AUC compared with healthy normal weight controls. Interestingly, these findings in adult women are similar to

Table 4 Relationship between leptin secretion and eating disorder psychopathology across groups. The correlation of EDI2 and EDE-Q scores with leptin pulsatile and basal secretion is presented. Spearman's correlation was used given the nonparametric distribution of leptin pulsatile and basal secretion.

\begin{tabular}{|c|c|c|c|c|}
\hline & \multicolumn{2}{|c|}{$\begin{array}{l}\text { Total pulsatile } \\
\text { secretion }\end{array}$} & \multicolumn{2}{|c|}{$\begin{array}{l}\text { Total basal } \\
\text { secretion }\end{array}$} \\
\hline & $R$ & $P$ & $R$ & $P$ \\
\hline Drive for thinness & -0.39 & 0.02 & -0.32 & 0.06 \\
\hline Bulimia & -0.31 & 0.08 & -0.35 & 0.04 \\
\hline Body dissatisfaction & -0.23 & 0.20 & -0.13 & 0.46 \\
\hline Ineffectiveness & -0.48 & 0.004 & -0.41 & 0.02 \\
\hline Perfectionism & -0.28 & 0.12 & -0.18 & 0.32 \\
\hline Interpersonal distrust & -0.37 & 0.03 & -0.20 & 0.25 \\
\hline Interoceptive awareness & -0.41 & 0.02 & -0.34 & 0.05 \\
\hline Maturity fears & -0.34 & 0.06 & -0.13 & 0.47 \\
\hline Asceticism & -0.52 & 0.002 & -0.39 & 0.02 \\
\hline Impulse regulation & -0.34 & 0.05 & -0.25 & 0.15 \\
\hline $\begin{array}{l}\text { Social insecurity } \\
\text { EDE-Q }\end{array}$ & -0.45 & 0.008 & -0.36 & 0.03 \\
\hline Dietary restraint & -0.43 & 0.01 & -0.47 & 0.005 \\
\hline Eating concern & -0.64 & $<0.0001$ & -0.23 & 0.19 \\
\hline Shape concern & -0.43 & 0.01 & -0.22 & 0.20 \\
\hline Weight concern & -0.38 & 0.03 & -0.30 & 0.09 \\
\hline Global score & -0.46 & 0.006 & -0.32 & 0.06 \\
\hline
\end{tabular}


our prior findings in adolescent girls with $\mathrm{AN}$ in whom one would expect the impact of pubertal status on the endocrine mileu (11). BASAL did not differ in AN women compared with normal weight controls, which suggests that lower leptin levels in AN women are a consequence of decreased TPS. Further, leptin half life and other specific leptin pulse characteristics that might impact TPS such as pulse frequency and pulse interval did not differ between groups, while the groups did differ for leptin pulse mass and amplitude. This indicates that decreased TPS and therefore total leptin secretion and AUC in the AN group is likely a result of reduced leptin pulse mass and pulse amplitude.

In contrast to the findings in $\mathrm{AN}$ women, $\mathrm{OB}$ women demonstrated increased leptin pulse amplitude, pulse mass, TPS and AUC compared with healthy normal weight controls. Similar to the AN group, OB women did not differ from controls in the number of pulses, interval between pulses or the half life of leptin. Our findings are consistent with those of Yildiz et al. (33), who compared the leptin secretory dynamics in lean and OB healthy men using cluster analysis and found no differences in pulse frequency or inter-peak interval between the two groups. In addition, in this study obese men had a significantly higher pulse amplitude compared with lean controls, similar to our findings. These data lead us to propose that leptin pulsatility is preserved in OB subjects, as was also seen in an early pilot study comparing leptin dynamics in one obese woman compared with a normal weight woman (34). Another study comparing women with PCOS with normally menstruating BMI matched controls using deconvolution analysis did not demonstrate any difference in the pulse frequency or amplitude. This study also had a low secretory burst mass $(5.37 \pm 1.47$ (controls) vs $8.31 \pm 2.90 \mathrm{ng} / \mathrm{ml}$ (PCOS)) compared with ours. However, this study yielded a calculated half life $(262 \pm 34.5$ (controls) vs $313 \pm 64.5 \mathrm{~min}$ (PCOS)) that was much higher than most studies, which might have impacted the findings (13). Additionally androgens are known to suppress leptin secretion (12), and therefore PCOS-associated hyperandrogenemia could account for the relatively low amplitude pulses in the PCOS women in this study. Although, it is well known that levels of leptin are elevated in OB subjects secondary to leptin resistance, our mechanistic data demonstrate that increased pulse amplitude and mass underlie the increased leptin secretion in overweight/obesity. Another important finding our study highlights is that leptin pulsatile secretion contributes to AUC, a measure of integrated leptin concentration. When we performed multivariate analysis controlling for BASAL, TPS emerged as the major predictor for leptin AUC.

When we examined associations of leptin secretory parameters with appetite regulating hormones, we found that TPS (but not BASAL) was inversely associated with ghrelin and cortisol. Ghrelin, produced in the stomach, and leptin have reciprocal effects on hypothalamic appetite pathways to produce orexigenic and anorexigenic effects respectively (35). Our earlier study in adolescents with AN and others have reported an inverse relationship between fasting levels of ghrelin and leptin $(8,10,11)$. Consistent with this, we found that TPS was also inversely correlated with fasting ghrelin levels. The association between leptin and cortisol, however, is less clear. Patients with Cushing's disease have high leptin levels (36) and glucocorticoids induce leptin secretion in vivo (37), suggesting a positive association between these hormones. In contrast, leptin has been shown to inhibit cortisol secretion in vitro (38) and in our earlier study in adolescents we reported a negative association between leptin pulsatile secretion and cortisol AUC and total cortisol secretion (11), consistent with the current findings. However, as seen in our earlier study, when percent body fat was included in the multivariate model, there were no significant associations between leptin characteristics and cortisol (11).

PYY is secreted peripherally in the intestine in response to food intake and acts on neuropeptide $\mathrm{Y}$ in the hypothalamus to induce satiety $(39,40)$. Although both leptin and PYY are anorexigenic in nature, patients with AN have high PYY and low leptin levels (17). Likewise, obese subjects also share an inverse relationship between PYY and leptin levels (41). TPS showed a similar inverse relationship to fasting PYY levels in our study subjects, which persisted after controlling for BASAL.

We found strong associations between both TPS and BASAL leptin and fasting insulin levels, which may reflect the established role of insulin in inducing leptin secretion $(6,30)$. We also report positive associations between leptin TPS and IGF1, which is consistent with prior studies showing relationships between levels of these nutritionally regulated hormones in both low and high weight states $(42,43)$. In our study, leptin TPS was positively associated with levels of $\mathrm{E}_{2}$, as previously shown in adolescents with AN (11) and healthy women (44), likely secondary to the positive influence of leptin on GnRH secretion. However, associations between leptin and other hormones were no longer significant after controlling for percent body fat, suggesting that underlying nutritional status may drive these correlations. The strong 
interrelationship between percent body fat and leptin as well as other endocrine factors makes it difficult to differentiate between the impact of body fat vs other hormonal factors on regulation of leptin secretion. Future interventional studies evaluating leptin secretory dynamics following nutritional and hormonal stimuli are warranted.

Low fasting leptin levels were associated with severity of disordered eating psychopathology in studies conducted in patients with AN $(17,45)$, while one other study showed contrasting findings (46). In our earlier study, we reported negative associations of fasting leptin levels with ineffectiveness, interoceptive awareness, interpersonal distrust and ascetism (17). The association of low fasting leptin levels with avoidance of sexual relationship measured by ascetism in EDI- 2 has also been demonstrated (45). However, leptin secretory dynamics were not evaluated in these studies. We now show that the leptin TPS is negatively associated with EDI-2 measures of asceticism, ineffectiveness and social insecurity and EDE$Q$ eating concern, and these relationships remain significant after controlling for BASAL. However, as seen with hormonal parameters, the associations of leptin TPS with eating disorder measures were no longer significant after controlling for percent body fat, suggesting that these associations may be consequent to underlying nutritional status and body fat.

Because leptin is secreted by adipocytes, percent body fat is undoubtedly the single major determinant of leptin concentrations. However, our findings go a step further and show that pulsatile rather than basal leptin secretion impacts leptin concentrations (AUC). At this time, our understanding of factors that drive basal vs pulsatile secretion of leptin is limited. Further studies exploring the impact of pulsatile vs basal secretion on disordered eating behavior are necessary.

It is difficult to attribute a causal relationship to the findings we demonstrate here given the cross sectional nature of this study. Although there is a relatively small number of subjects in each of the three groups, our sample size compares well with other studies that have used deconvolution analysis for evaluating leptin secretory dynamics $(33,34)$ as well as those reporting psychopathology measures $(47,48)$. Additionally, the ability to detect significant differences in this sample size indicates the strength of our findings. All women with AN in our study were low weight. A future direction would be to examine changes in leptin secretory dynamics with weight recovery.

\section{Conclusion}

Secretory dynamics of leptin vary notably across the weight spectrum. Pulsatile leptin secretion makes a significant contribution to total circulating leptin levels and is associated with levels of other appetite regulating hormones and disordered eating psychopathology, possibly reflecting nutritional status and proportion of body fat. To better understand these associations, further investigations exploring the role of pulsatile vs basal leptin secretion in regulation of disordered eating are warranted.

\section{Declaration of interest}

All authors declare that there is no conflict of interest that could be perceived as prejudicing the impartiality of the research reported.

\section{Funding}

This study was supported by funding from the following sources: Investigator initiated grant from Bioenvision, New York, NY; National Institutes of Health Grant K23 MH092560, Bethesda, MD; Harvard Catalyst, the Harvard Clinical and Translational Science Center UL1 RR025758, Boston, MA; Training grant T32 DK007028, NIH, Bethesda, MD.

\section{Acknowledgements}

We sincerely thank $\mathrm{Dr}$ Anne Klibanski MD, Neuroendocrine Unit, Massachusetts General Hospital and Harvard Medical School, Boston, MA, USA for her critical review of the manuscript, the staff at the clinical research center, and study participants.

\section{References}

1 Sinha MK, Sturis J, Ohannesian J, Magosin S, Stephens T, Heiman ML, Polonsky KS \& Caro JF. Ultradian oscillations of leptin secretion in humans. Biochemical and Biophysical Research Communications 1996228 733-738. (doi:10.1006/bbrc.1996.1724)

2 Fors H, Matsuoka H, Bosaeus I, Rosberg S, Wikland KA \& Bjarnason R. Serum leptin levels correlate with growth hormone secretion and body fat in children. Journal of Clinical Endocrinology and Metabolism 199984 3586-3590. (doi:10.1210/jcem.84.10.6035)

3 Grinspoon S, Gulick T, Askari H, Landt M, Lee K, Anderson E, Ma Z, Vignati L, Bowsher R, Herzog D et al. Serum leptin levels in women with anorexia nervosa. Journal of Clinical Endocrinology and Metabolism 1996 81 3861-3863. (doi:10.1210/jcem.81.11.8923829)

4 Silha JV, Krsek M, Skrha JV, Sucharda P, Nyomba BL \& Murphy LJ. Plasma resistin, adiponectin and leptin levels in lean and obese subjects: correlations with insulin resistance. European Journal of Endocrinology 2003149 331-335. (doi:10.1530/eje.0.1490331)

5 Schoeller DA, Cella LK, Sinha MK \& Caro JF. Entrainment of the diurnal rhythm of plasma leptin to meal timing. Journal of Clinical Investigation 1997100 1882-1887. (doi:10.1172/JCI119717)

6 Cammisotto PG, Gelinas Y, Deshaies Y \& Bukowiecki LJ. Regulation of leptin secretion from white adipocytes by insulin, glycolytic substrates, and amino acids. American Journal of Physiology. Endocrinology and Metabolism 2005289 E166-E171. (doi:10.1152/ajpendo.00602.2004) 
7 Cammisotto PG \& Bendayan M. Leptin secretion by white adipose tissue and gastric mucosa. Histology and Histopathology 200722 199-210.

8 Tolle V, Kadem M, Bluet-Pajot MT, Frere D, Foulon C, Bossu C, Dardennes R, Mounier C, Zizzari P, Lang F et al. Balance in ghrelin and leptin plasma levels in anorexia nervosa patients and constitutionally thin women. Journal of Clinical Endocrinology and Metabolism $2003 \mathbf{8 8}$ 109-116. (doi:10.1210/jc.2002-020645)

9 Elimam A, Knutsson U, Bronnegard M, Stierna P, Albertsson-Wikland K $\&$ Marcus C. Variations in glucocorticoid levels within the physiological range affect plasma leptin levels. European Journal of Endocrinology 1998139 615-620. (doi:10.1530/eje.0.1390615)

10 Germain N, Galusca B, Le Roux CW, Bossu C, Ghatei MA, Lang F, Bloom SR \& Estour B. Constitutional thinness and lean anorexia nervosa display opposite concentrations of peptide YY, glucagon-like peptide 1, ghrelin, and leptin. American Journal of Clinical Nutrition 2007 85 967-971.

11 Misra M, Miller KK, Kuo K, Griffin K, Stewart V, Hunter E, Herzog DB \& Klibanski A. Secretory dynamics of leptin in adolescent girls with anorexia nervosa and healthy adolescents. American Journal of Physiology. Endocrinology and Metabolism 2005289 E373-E381. (doi:10.1152/ajpendo.00041.2005)

12 Blum WF, Englaro P, Hanitsch S, Juul A, Hertel NT, Muller J, Skakkebaek NE, Heiman ML, Birkett M, Attanasio AM et al. Plasma leptin levels in healthy children and adolescents: dependence on body mass index, body fat mass, gender, pubertal stage, and testosterone. Journal of Clinical Endocrinology and Metabolism 199782 2904-2910. (doi:10.1210/jcem.82.9.4251)

13 Iuorno MJ, Islam LZ, Veldhuis PP, Boyd DG, Farhy LS, Johnson ML, Nestler JE \& Evans WS. Leptin secretory burst mass correlates with body mass index and insulin in normal women but not in women with polycystic ovary syndrome. Metabolism 200756 1561-1565. (doi:10.1016/j.metabol.2007.06.027)

14 Brzechffa PR, Jakimiuk AJ, Agarwal SK, Weitsman SR, Buyalos RP \& Magoffin DA. Serum immunoreactive leptin concentrations in women with polycystic ovary syndrome. Journal of Clinical Endocrinology and Metabolism 199681 4166-4169. (doi:10.1210/jcem.81.11.8923878)

15 Remsberg KE, Talbott EO, Zborowski JV, Evans RW \& McHugh-Pemu K. Evidence for competing effects of body mass, hyperinsulinemia, insulin resistance, and androgens on leptin levels among lean, overweight, and obese women with polycystic ovary syndrome. Fertility and Sterility 200278 479-486. (doi:10.1016/S0015-0282(02)03303-4)

16 Adami G, Campostano A, Cella F \& Ferrandes G. Serum leptin level and restrained eating: study with the Eating Disorder Examination. Physiology \& Behavior 200275 189-192. (doi:10.1016/S00319384(01)00639-4)

17 Lawson EA, Eddy KT, Donoho D, Misra M, Miller KK, Meenaghan E, Lydecker J, Herzog D \& Klibanski A. Appetite-regulating hormones cortisol and peptide YY are associated with disordered eating psychopathology, independent of body mass index. European Journal of Endocrinology 2011164 253-261. (doi:10.1530/EJE-10-0523)

18 Lawson EA, Donoho D, Miller KK, Misra M, Meenaghan E, Lydecker J, Wexler T, Herzog DB \& Klibanski A. Hypercortisolemia is associated with severity of bone loss and depression in hypothalamic amenorrhea and anorexia nervosa. Journal of Clinical Endocrinology and Metabolism 200994 4710-4716. (doi:10.1210/jc.2009-1046)

19 Lawson EA, Miller KK, Blum JI, Meenaghan E, Misra M, Eddy KT, Herzog DB \& Klibanski A. Leptin levels are associated with decreased depressive symptoms in women across the weight spectrum, independent of body fat. Clinical Endocrinology 201276 520-525. (doi:10.1111/ j.1365-2265.2011.04182.x)

20 Lawson EA, Donoho DA, Blum JI, Meenaghan EM, Misra M, Herzog DB, Sluss PM, Miller KK \& Klibanski A. Decreased nocturnal oxytocin levels in anorexia nervosa are associated with low bone mineral density and fat mass. Journal of Clinical Psychiatry 201172 1546-1551. (doi:10.4088/ JCP.10m06617)
21 Eddy KT, Lawson EA, Meade C, Meenaghan E, Horton SE, Misra M, Klibanski A \& Miller KK. Appetite regulatory hormones in women with anorexia nervosa: binge-eating/purging versus restricting type. Journal of Clinical Psychiatry 201576 19-24. (doi:10.4088/JCP. $13 \mathrm{~m} 08753)$

22 Veldhuis JD \& Johnson ML. Deconvolution analysis of hormone data. Methods in Enzymology 1992210 539-575. (doi:10.1016/00766879(92)10028-C)

23 Klein S, Coppack SW, Mohamed-Ali V \& Landt M. Adipose tissue leptin production and plasma leptin kinetics in humans. Diabetes 199645 984-987. (doi:10.2337/diab.45.7.984)

24 Fairburn CG, Cooper Z, Marianne O, Ed. The eating disorder examination. In Binge Eating: Nature, Assessment and Treatment. New York: Guilford Press, 1993.

25 Mond JM, Hay PJ, Rodgers B \& Owen C. Eating Disorder Examination Questionnaire (EDE-Q): norms for young adult women. Behaviour Research and Therapy 200644 53-62. (doi:10.1016/j.brat.2004.12.003)

26 Luce KH, Crowther JH \& Pole M. Eating Disorder Examination Questionnaire (EDE-Q): norms for undergraduate women. International Journal of Eating Disorders 200841 273-276. (doi:10.1002/eat.20504)

27 Garner DM. Eating Disorders Inventory-2: Professional Manual. Odessa, FL: Psychological Assessment Resources Inc, 1991.

28 Savgan-Gurol E, Bredella M, Russell M, Mendes N, Klibanski A \& Misra M. Waist to hip ratio and trunk to extremity fat (DXA) are better surrogates for IMCL and for visceral fat respectively than for subcutaneous fat in adolescent girls. Nutrition and Metabolism 20107 86. (doi:10.1186/1743-7075-7-86)

29 Ackerman KE, Davis B, Jacoby L \& Misra M. DXA surrogates for visceral fat are inversely associated with bone density measures in adolescent athletes with menstrual dysfunction. Journal of Pediatric Endocrinology \& Metabolism 201124 497-504. (doi:10.1515/jpem.2011.208)

30 Kolaczynski JW, Nyce MR, Considine RV, Boden G, Nolan JJ, Henry R, Mudaliar SR, Olefsky J \& Caro JF. Acute and chronic effects of insulin on leptin production in humans: studies in vivo and in vitro. Diabetes 1996 45 699-701. (doi:10.2337/diab.45.5.699)

31 Misra M \& Klibanski A. Endocrine consequences of anorexia nervosa. Lancet. Diabetes \& Endocrinology 20142 581-592. (doi:10.1016/ S2213-8587(13)70180-3)

32 Saad MF, Damani S, Gingerich RL, Riad-Gabriel MG, Khan A, Boyadjian R, Jinagouda SD, el-Tawil K, Rude RK \& Kamdar V. Sexual dimorphism in plasma leptin concentration. Journal of Clinical Endocrinology and Metabolism 199782 579-584. (doi:10.1210/jcem.82. 2.3739)

33 Yildiz BO, Suchard MA, Wong ML, McCann SM \& Licinio J. Alterations in the dynamics of circulating ghrelin, adiponectin, and leptin in human obesity. PNAS 2004101 10434-10439. (doi:10.1073/pnas. 0403465101)

34 Licinio J, Mantzoros C, Negrao AB, Cizza G, Wong ML, Bongiorno PB, Chrousos GP, Karp B, Allen C, Flier JS et al. Human leptin levels are pulsatile and inversely related to pituitary-adrenal function. Nature Medicine 19973 575-579. (doi:10.1038/nm0597-575)

35 Klok MD, Jakobsdottir S \& Drent ML. The role of leptin and ghrelin in the regulation of food intake and body weight in humans: a review. Obesity Reviews 20078 21-34. (doi:10.1111/j.1467-789X.2006.00270.x)

36 Robaczyk M, Krzyzanowiska-Swiniarska B, Andrysiak-Mamos E, Kulig G \& Pilarska K. Plasma leptin levels in relation to body composition and body fat distribution in patients with Cushing's syndrome. Polskie Archiwum Medycyny Wewneçtrznej 2003110 1299-1308.

37 Papaspyrou-Rao S, Schneider SH, Petersen RN \& Fried SK. Dexamethasone increases leptin expression in humans in vivo. Journal of Clinical Endocrinology and Metabolism 199782 1635-1637. (doi:10.1210/jcem.82.5.3928)

38 Glasow A, Haidan A, Hilbers U, Breidert M, Gillespie J, Scherbaum WA, Chrousos GP \& Bornstein SR. Expression of Ob receptor in normal human adrenals: differential regulation of adrenocortical and 
adrenomedullary function by leptin. Journal of Clinical Endocrinology and Metabolism 199883 4459-4466. (doi:10.1210/jcem.83.12.5337)

39 Adrian TE, Ferri GL, Bacarese-Hamilton AJ, Fuessl HS, Polak JM \& Bloom SR. Human distribution and release of a putative new gut hormone, peptide YY. Gastroenterology 198589 1070-1077.

40 Renshaw D, Batterham RL \& Peptide YY. a potential therapy for obesity. Current Drug Targets 20056 171-179. (doi:10.2174/ 1389450053174523)

41 Batterham RL, Cohen MA, Ellis SM, Le Roux CW, Withers DJ, Frost GS, Ghatei MA \& Bloom SR. Inhibition of food intake in obese subjects by peptide YY3-36. New England Journal of Medicine 2003349 941-948. (doi:10.1056/NEJMoa030204)

42 Haspolat K, Ece A, Gurkan F, Atamer Y, Tutanc M \& Yolbas I. Relationships between leptin, insulin, IGF-1 and IGFBP-3 in children with energy malnutrition. Clinical Biochemistry 200740 201-205. (doi:10.1016/j.clinbiochem.2006.11.008)

43 Zarghami NM \& Karimi P. Relationship between insulin like growth factor-1 and leptin in type II diabetic patients. International Journal of Endocrinology and Metabolism 19961 26-34.

44 Licinio J, Negrao AB, Mantzoros C, Kaklamani V, Wong ML, Bongiorno PB, Mulla A, Cearnal L, Veldhuis JD, Flier JS et al. Synchronicity of frequently sampled, 24-h concentrations of circulating leptin, luteinizing hormone, and estradiol in healthy women. PNAS 199895 2541-2546. (doi:10.1073/pnas.95.5.2541) 45 Ehrlich S, Burghardt R, Schneider N, Hein J, Weiss D, Pfeiffer E, Lehmkuhl U \& Salbach-Andrae H. Leptin and its associations with measures of psychopathology in patients with anorexia nervosa. Journal of Neural Transmission 2009116 109-115. (doi:10.1007/s00702008-0151-3)

46 Monteleone P, Di Lieto A, Tortorella A, Longobardi N \& Maj M. Circulating leptin in patients with anorexia nervosa, bulimia nervosa or binge-eating disorder: relationship to body weight, eating patterns, psychopathology and endocrine changes. Psychiatry Research 200094 121-129. (doi:10.1016/S0165-1781(00)00144-X)

47 Bomba M, Gambera A, Bonini L, Peroni M, Neri F, Scagliola P \& Nacinovich R. Endocrine profiles and neuropsychologic correlates of functional hypothalamic amenorrhea in adolescents. Fertility and Sterility 200787 876-885. (doi:10.1016/j.fertnstert.2006.09.011)

48 Lawson EA, Holsen LM, Santin M, Meenaghan E, Eddy KT, Becker AE, Herzog DB, Goldstein JM \& Klibanski A. Oxytocin secretion is associated with severity of disordered eating psychopathology and insular cortex hypoactivation in anorexia nervosa. Journal of Clinical Endocrinology and Metabolism 201297 E1898-E1908. (doi:10.1210/ jc.2012-1702)

Received 28 August 2015

Revised version received 11 December 2015

Accepted 18 January 2016 